ESAIM: PROCEEDINGS AND SURVEYS, April 2020, Vol. 67, p. 1-15

Vincent Calvez, Céline Grandmont, Eva Locherbach, Clair Poignard, Magali Ribot, Nicolas Vauchelet Editors

\title{
PHASE TRANSITIONS IN A TWO-SPECIES MODEL FOR CELL SEGREGATION AND LOGISTIC GROWTH
}

\author{
Luis Almeida ${ }^{1}$, Kevin Atsou ${ }^{2}$, Marta Marulli ${ }^{3}$, Diane Peurichard ${ }^{4}$ and Rémi \\ TESSON $^{5}$
}

\begin{abstract}
We study a model of cell segregation in a population composed of two cell types. Starting from a model initially proposed in 3, we aim to understand the impact of a cell division process on the system's segregation abilities. The original model describes a population of spherical cells interacting with their close neighbors by means of a repulsion potential and which centers are subject to Brownian motion. Here, we add a stochastic birth-death process in the agent-based model, that approaches a logistic growth term in the continuum limit. We address the linear stability of the spatially homogeneous steady states of the macroscopic model and obtain a precise criterion for the phase transition, which links the system segregation ability to the model parameters. By comparing the criterion with the one obtained without logistic growth, we show that the system's segregation ability is the result of a complex interplay between logistic growth, diffusion and mechanical repulsive interactions. Numerical simulations are presented to illustrate the results obtained at the microscopic scale.

Résumé. Nous étudions un modèle de ségrégation cellulaire dans une population composée de deux types de cellules. En partant d'un modèle initialement proposé dans 3, nous cherchons à comprendre l'impact d'une division cellulaire sur les capacités de ségrégation du système. Le modèle original décrit une population de cellules sphériques interagissant avec leurs voisins proches par le biais d'un potentiel de répulsion et dont les centres sont soumis à un mouvement brownien. Ici, nous ajoutons un processus stochastique de naissance et de mort dans le modèle à agents, qui approche un terme de croissance logistique dans la limite du continu. Nous étudions la stabilité linéaire des états stationnaires spatialement homogènes du modèle macroscopique et nous obtenons un critère précis pour la transition de phase, qui lie la capacité de ségrégation du système aux paramètres du modèle. En comparant le critère avec celui obtenu sans croissance logistique, nous montrons que la capacité de ségrégation du système est le résultat d'une interaction complexe entre croissance logistique, diffusion et interactions mécaniques répulsives. Des simulations numériques sont présentés pour illustrer les résultats obtenus à l'échelle microscopique.
\end{abstract}

\footnotetext{
${ }^{1}$ Sorbonne Université, CNRS, Laboratoire Jacques-Louis-Lions, 4 place Jussieu, 75005, Paris, France

2 INRIA, Laboratoire J.A. Dieudonné, Université de Nice Sophia-Antipolis,

${ }^{3}$ LAGA, UMR 7539, CNRS, Université Paris 13, France, University of Bologna, Italy

${ }^{4}$ Sorbonne Université, Inria, Mamba project-team, Laboratoire Jacques-Louis Lions, 4 place Jussieu, 75005, Paris, France.

${ }^{5}$ Aix Marseille Univ, CNRS, Centrale Marseille, I2M, Marseille, France.
} 
ESAIM: PROCEEDINGS AND SURVEYS

\section{INTRODUCTION}

The starting point of this work was the model previously proposed in [1], 2. The authors provided a detailed multiscale analysis - from a microscopic model to a macroscopic description - of a system of particles interacting through a dynamical network. The model describes point particles with local cross-links modeled by springs that are randomly created and destructed. Each link between two particles generates a springlike interaction potential, which depends on the link type (intra- or inter- species link). In the limit of large number of particles and links and assuming that the network remodelling rate is very large, the link density distribution becomes completely determined by the one-particle distribution function. The latter evolves on the slow time scale through an aggregation-diffusion equation, also known as the McKean-Vlasov equation. Their results have been extended and applied to the two-species case in order to study segregation mechanisms in tissue morphogenesis 3. The ability of different cell types to segregate is known to be a key process in many biological phenomena, especially in embryogenesis or tumor metastasis. However, in their model, it was assumed that the cell population remains constant over time, which means that there is no growth process. The goal of this paper is to investigate whether cell growth processes can enhance or knock out the system's segregation abilities, when cell segregation is otherwise driven by mechanical interactions between different types of cells. To this aim, we derive a macroscopic logistic equation from the microscopic two-species model introduced in [3], modified to take into consideration a density-saturated growth process at the microscopic scale. We will focus on the influence that homotypic/heterotypic repulsion has on the process of cell segregation and border sharpening, inspired from the results in 6 . After the derivation of the macroscopic model we carry out its stability analysis and we perform numerical simulations of the microscopic model.

\section{THE MICROSCOPIC MODEL}

We describe the microscopic model for cells belonging to two distinct species with an additional growth process. The model introduced in [3], is a 2D individual based model which depicts two species of cells referred to as type A and type B cells. The cells are represented by $2 \mathrm{D}$-spheres located by their centers and interacting with each others by creating and suppressing links via a random process with neighbouring cells located in a ball of radius $\mathrm{R}$ from their centers. The links creation and suppression are supposed to follow Poisson processes of frequencies $\nu_{c}^{A A}, \nu_{c}^{B B}, \nu_{c}^{A B}$ and $\nu_{d}^{A A}, \nu_{d}^{B B}, \nu_{d}^{A B}$, where the subscripts $c$ and $d$ denote respectively 'creation' and 'deletion' and the superscripts denote respectively the intraspecies links $(A A, B B)$ and interspecies links $(A B)$. Once created, the links generate a spring-like mechanical potential which depends on the type of interactions (Figure 1). For instance in order to account for the effect of repulsion in the segregation phenomenon, the amplitude of the potential generated by inter-species (heterotypic) links might be greater than the one generated

by intra-species(homotypic) links. Each cell is supposed to be animated by a two dimensional brownian motion in order to describe its movement inside the tissue. 


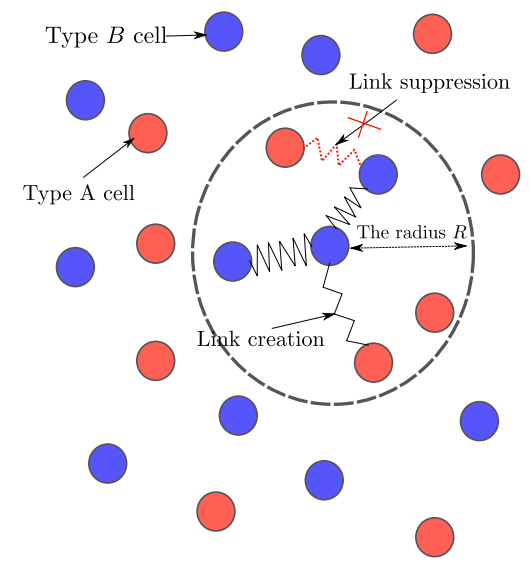

FiguRE 1. Illustration of the cells interacting through the network of links

We stress the fact that the effects due to cell deformations are neglected. The description of the model is restricted to two spatial dimensions. The set of type A and type B cells contains respectively $N_{A}$ and $N_{B}$ individuals and the dynamic of their centers are described by points $\left(X_{i}^{A}, X_{l}^{B}\right) \in \mathbb{R}^{2} \times \mathbb{R}^{2}, i \in\left\{1, \cdots, N_{A}\right\}, l \in$ $\left\{1, \cdots, N_{B}\right\}$. The intra-species links generate potentials $\Phi^{A A}\left(X_{i}^{A}, X_{j}^{A}\right)$ and $\Phi^{B B}\left(X_{l}^{B}, X_{m}^{B}\right)$ and interspecies links generate potentials $\Phi^{A B}\left(X_{i}^{A}, X_{l}^{B}\right)$ and $\Phi^{B A}\left(X_{l}^{B}, X_{i}^{A}\right)$ not necessarily symmetric. Those potentials incorporate the fact that the two particle families act differently on each other. Hence, particle motion between two particles linking-unlinking events is supposed to occur in the steepest gradient descent of the total mechanical potential:

$$
\begin{cases}d X_{i}^{A}=-\mu \nabla_{X_{i}^{A}} W^{A}\left(X^{A}, X^{B}\right) d t+\sqrt{2 D_{A}} d \mathcal{B}_{i}, \quad \forall i \in\left\{1, \ldots, N_{A}\right\} \\ d X_{i}^{B}=-\mu \nabla_{X_{i}^{A}} W^{B}\left(X^{A}, X^{B}\right) d t+\sqrt{2 D_{B}} d \mathcal{B}_{i}, \quad \forall i \in\left\{1, \ldots, N_{B}\right\}\end{cases}
$$

where $\mu>0$ is the mobility coefficient considered to be given and $\mathcal{B}_{i}$ is a 2-dimensional Brownian motion $\mathcal{B}_{i}=\left(\mathcal{B}_{i}^{1} ; \mathcal{B}_{i}^{2}\right)$ with intensity $D_{A}>0$ for specie A and $D_{B}>0$ for species B. We define $W^{S}$ the total potential of the type-S particle, $S \in\{A, B\}$, as the sum over all pairwise link potentials acting on particles S:

$$
W^{S}\left(X^{S}, X^{T}\right)=\sum_{k_{1}=1}^{K_{S S}} \Phi^{S S}\left(X_{i\left(k_{1}\right)}^{S}, X_{j\left(k_{1}\right)}^{S}\right)+\sum_{k_{3}=1}^{K_{S T}} \Phi^{S T}\left(X_{m\left(k_{3}\right)}^{S}, X_{l\left(k_{3}\right)}^{T}\right),
$$

where $k_{1}$ and $k_{3}$ refer to the indexes of intra-species link and inter-species link, respectively, and where each link $k_{1}$ (resp. $\left.k_{3}\right)$ is associated with a unique pair of cell indexes $\left(i\left(k_{1}\right), j\left(k_{1}\right)\right)\left(\left(m\left(k_{3}\right), l\left(k_{3}\right)\right)\right.$ resp. $)$. The potential $\Phi^{S T}$ refers to the action a type $T$ particle exerts on a type $S$ particle while $\Phi^{T S}$ is the action a type $S$ particle exerts on a particle of type $T$. All along the paper, we will consider Hookean-type potentials of the form:

$$
\Phi^{S T}\left(x_{1}, x_{2}\right)=\frac{\kappa^{S T}}{2}\left(\left|x_{1}-x_{2}\right|-R\right)^{2} .
$$

The main extension in the model is the introduction of a cell birth and death process. Our modeling is based on the birth and death process proposed in 7 . The idea is that a cell of the type $S$ population has a probability $\beta_{S}$ to divide into two cells and a probability $\delta_{S}$ to die at each time step. To introduce the spatial logistic effect at the microscopic scale, we assume that the birth and death processes depend on the local density of individuals divided by the local carrying capacity of the population:

$$
\beta_{S}\left(X_{i}^{S}\right)=b_{0}^{S}-\left(b_{0}^{S}-\theta_{S}\right)\left(\frac{\mathcal{N}_{R_{0}}\left(X_{i}^{S}\right)}{N^{*}}\right), \quad \delta_{S}\left(X_{i}^{S}\right)=d_{0}^{S}+\left(\theta_{S}-d_{0}^{S}\right)\left(\frac{\mathcal{N}_{R_{0}}\left(X_{i}^{S}\right)}{N^{*}}\right)
$$


where the coefficient $\mathcal{N}_{R_{0}}\left(X_{i}^{S}\right)$ is the number of cells (of both population) which centers are located at distance $R_{0}$ of $X_{i}^{S}$ and $N^{*}$ is the target number of cells in a ball of radius $R_{0}$ and is referred to as the local carrying capacity. The parameters $b_{0}^{S}$ and $d_{0}^{S}$ are respectively the intrinsic birth rate and death rate of an individual, the parameter $\theta_{S}$ is the turnover, which is equal to the birth and death probabilities when the population reaches its local population carrying capacity $N^{*}$. Note that $\theta_{S}$ must be taken in the range $d_{0}^{S}<\theta_{S}<b_{0}^{S}$. The probability for a cell to give birth or die within a small time step $\tau$ is respectively (see section 'Numerical results' for more details on the process):

$$
\tau \beta\left(X_{i}^{S}\right) \text { or } \tau \delta\left(X_{i}^{S}\right)
$$

Remark Such a birth and death process has been shown to approach (in the limit of large number of particles) a logistic equation, where the deterministic population growth rate is $b_{0}-d_{0}$ (see [7] and references therein). Therefore, the condition $d_{0}<b_{0}$ is introduced to ensure the positivity of the deterministic growth rate.

\section{THE DERIVATION OF THE MACROSCOPIC MODEL}

We study the main steps needed to perform the derivation of the macroscopic model from the microscopic dynamics. Following 3], we are interested in a regime of fast linking-unlinking of the particles. To this aim, we introduce an intermediate microscopic model in which the limit of fast linking/unlinking is considered for fixed number of cells, and then derive the PDE associated with this microscopic dynamics.

\subsection{The microscopic dynamics in the limit of fast linking/unlinking processes}

We denote by $A_{i j}(t), B_{i j}(t), C_{i j}(t)$ the adjacency matrices of particles $A, B$, and cross-links $A-B$ respectively. In particular, for $i, j \in\left\{1, \ldots, N_{A}\right\}, A_{i j}(t)=1$ (resp. =0) if particles of type $A i$ and $j$ are connected at time $t$ (resp. not connected). The definition of matrix $B$ is similar. For $i \in\left\{1, \ldots, N_{A}\right\}, j \in\left\{1, \ldots, N_{B}\right\}, C_{i j}(t)=1$ (resp. $=0)$ if particle $i$ of type $A$ and particle $j$ of type $B$ are connected at time $t$ (resp. not connected). $A$ and $B$ are square symmetric matrices, and $C$ is an $N_{A} \times N_{B}$ rectangular matrix.

The derivation of the reduced microscopic model relies on averaging. The particles positions $X_{i}^{A}, X_{j}^{B}(t)$ are slow processes, and the links $A_{i j}(t), B_{i j}(t), C_{i j}(t)$ are fast processes: they quickly converge to stationary measures, which depends on the $X_{i}^{A, B}(t)$. We will then compute the evolution of $X_{i}^{A, B}$ by averaging the basic dynamical equation (1) over these stationary measures of the links processes.

The process for the links writes

$$
\begin{aligned}
d A_{i j}(t) & =-A_{i j}(t) d N_{i j}^{A A, d}(t)+\left[1-A_{i j}(t)\right] \chi_{\left|X_{i}^{A}(t)-X_{j}^{A}(t)\right| \leq R} d N_{i j}^{A A, f}(t) \\
d B_{i j}(t) & =-B_{i j}(t) d N_{i j}^{B B, d}(t)+\left[1-B_{i j}(t)\right] \chi_{\left|X_{i}^{B}(t)-X_{j}^{B}(t)\right| \leq R} d N_{i j}^{B B, f}(t) \\
d C_{i j}(t) & =-C_{i j}(t) d N_{i j}^{A B, d}(t)+\left[1-C_{i j}(t)\right] \chi_{\left|X_{i}^{A}(t)-X_{j}^{B}(t)\right| \leq R} d N_{i j}^{A B, f}(t)
\end{aligned}
$$

where the $N_{i j}^{A A, d}, N_{i j}^{A A, f}, N_{i j}^{B B, d}, N_{i j}^{B B, f}, N_{i j}^{A B, d}, N_{i j}^{A B, f}$, are independent Poisson processes, with rates respectively

$$
\nu_{d}^{A A}=\tilde{\nu}_{d}^{A A} \varepsilon^{-2}, \nu_{c}^{A A}=\tilde{\nu}_{c}^{A A} \varepsilon^{-2}, \nu_{d}^{B B}=\tilde{\nu}_{d}^{B B} \varepsilon^{-2}, \nu_{c}^{B B}=\tilde{\nu}_{f}^{B B} \varepsilon^{-2}, \nu_{d}^{A B}=\tilde{\nu}_{d}^{A B} \varepsilon^{-2}, \nu_{c}^{A B}=\tilde{\nu}_{c}^{B B} \varepsilon^{-2}
$$


where $\epsilon \ll 1$. Conditionally on positions $X_{i}^{A}, X_{j}^{B}$, all the processes $A_{i j}, B_{i j}, C_{i j}$ are independent. The stationary measures of (5)-(6)-(7), for fixed positions $X_{i}^{A}, X_{j}^{B}$ are then simply product of Bernoulli measures:

$$
\begin{aligned}
& \mathbb{P}\left(A_{i j}=1\right)=\frac{\tilde{\nu}_{c}^{A A} \chi_{\left|X_{i}^{A}(t)-X_{j}^{A}(t)\right| \leq R}}{\tilde{\nu}_{c}^{A A}+\tilde{\nu}_{d}^{A A}} \quad, \quad \mathbb{P}\left(A_{i j}=0\right)=1-\mathbb{P}\left(A_{i j}=1\right) \\
& \mathbb{P}\left(B_{i j}=1\right)=\frac{\tilde{\nu}_{c}^{B B} \chi_{\left|X_{i}^{B}(t)-X_{j}^{B}(t)\right| \leq R}}{\tilde{\nu}_{c}^{B B}+\tilde{\nu}_{d}^{B B}} \quad, \quad \mathbb{P}\left(B_{i j}=0\right)=1-\mathbb{P}\left(B_{i j}=1\right) \\
& \mathbb{P}\left(C_{i j}=1\right)=\frac{\tilde{\nu}_{c}^{A B} \chi_{\left|X_{i}^{A}(t)-X_{j}^{B}(t)\right| \leq R}}{\tilde{\nu}_{c}^{A B}+\tilde{\nu}_{d}^{A B}} \quad, \quad \mathbb{P}\left(C_{i j}=0\right)=1-\mathbb{P}\left(C_{i j}=1\right) .
\end{aligned}
$$

Defining:

$$
\tilde{\Phi}^{S T}(x)=\frac{\kappa^{S T}}{2}\left\{\begin{array}{ll}
(|x|-R)^{2} & \text { for }|x| \leq R \\
0 & \text { for }|x|>R
\end{array},\right.
$$

one can write the equations for the positions, averaged over the stationary measure for the links:

$$
\begin{aligned}
d X_{i}^{A} & =-\mu\left(\frac{\tilde{\nu}_{c}^{A A}}{\tilde{\nu}_{c}^{A A}+\tilde{\nu}_{d}^{A A}} \sum_{j=1}^{N_{A}} \nabla \tilde{\Phi}^{A A}\left(X_{i}^{A}-X_{j}^{A}\right)+\frac{\tilde{\nu}_{c}^{A B}}{\tilde{\nu}_{c}^{A B}+\tilde{\nu}_{d}^{A B}} \sum_{j=1}^{N_{B}} \nabla \tilde{\Phi}^{A B}\left(X_{i}^{A}-X_{j}^{B}\right)\right) d t+\sqrt{2 D^{A}} d \mathcal{B}_{i}^{A}(8) \\
d X_{i}^{B} & =-\mu\left(\frac{\tilde{\nu}_{c}^{A B}}{\tilde{\nu}_{c}^{A B}+\tilde{\nu}_{d}^{A B}} \sum_{j=1}^{N_{A}} \nabla \tilde{\Phi}^{B A}\left(X_{i}^{B}-X_{j}^{A}\right)+\frac{\tilde{\nu}_{c}^{B B}}{\tilde{\nu}_{c}^{B B}+\tilde{\nu}_{d}^{B B}} \sum_{j=1}^{N_{B}} \nabla \tilde{\Phi}^{B B}\left(X_{i}^{B}-X_{j}^{B}\right)\right) d t+\sqrt{2 D^{B}} d \mathcal{B}_{i}^{B}(9)
\end{aligned}
$$

Notice that in this limit a particle interacts with all its neighbors at distance smaller than $R$ with intensity decreased by a factor $\frac{\tilde{\nu}_{c}^{S T}}{\tilde{\nu}_{c}^{S T}+\tilde{\nu}_{d}^{S T}} \leq 1$. Without loss of generality, we will now choose the time and space scales such that $\mu=1$.

\subsection{Macroscopic description of the intermediate model}

As we would like to analyze the microscopic dynamics from a macroscopic point of view, we aim to derive the PDE associated with the dynamics (8)-(9). The standard method is to consider the so-called empirical distributions $f^{A}(x, t), f^{B}(x, t)$ of the $N_{A}$ type-A and $N_{B}$ type-B cells respectively:

$$
f^{A}(x, t)=\sum_{i=1}^{N_{A}} \delta\left(x-X_{i}^{A}(t)\right), \quad f^{B}(x, t)=\sum_{i=1}^{N_{B}} \delta\left(x-X_{i}^{B}(t)\right),
$$

where $\left\{X_{i}^{A}(t), X_{i}^{B}(t)\right\}$ are solutions of the dynamical system (8)-(9). To find the equations satisfied by the empirical distributions, we integrate $f^{A}(x, t), f^{B}(x, t)$ against a test function $\phi$ and take the time derivatives. One deduces that if $b_{0}, d_{0}, \theta_{S}=0$ (without growth terms), $f^{A}(x, t), f^{B}(x, t)$ satisfie (weakly) the following equations:

$$
\begin{aligned}
& \partial_{t} f^{A}=D^{A} \Delta_{x} f^{A}+\nabla_{x} \cdot\left(f^{A} \nabla_{x}\left(\bar{\Phi}^{A A} * f^{A}\right)\right)+\nabla_{x} \cdot\left(f^{A} \nabla_{x}\left(\bar{\Phi}^{A B} * f^{B}\right)\right) \\
& \partial_{t} f^{B}=D^{B} \Delta_{x} f^{B}+\nabla_{x} \cdot\left(f^{B} \nabla_{x}\left(\bar{\Phi}^{B B} * f^{B}\right)\right)+\nabla_{x} \cdot\left(f^{B} \nabla_{x}\left(\bar{\Phi}^{B A} * f^{B}\right)\right),
\end{aligned}
$$

where the factors $\frac{\tilde{\nu}_{c}^{S T}}{\tilde{\nu}_{c}^{S T}+\tilde{\nu}_{d}^{S T}}$ have been included in the potential functions $\bar{\Phi}^{S T}$. Now, Eqs. 111 do not take into account growth phenomena. According to the description at the beginning of the paper, our model describes 
cell birth and death according to a logistic growth-like process: given a type-S cell ( $\mathrm{S}$ being either A or B) at position $x$, its probability $P^{S}(x)$ to give birth to a new cell depends on the number of neighboring cells (type-A or type-B) contained in a ball of center $x$ and radius $R_{0}$ :

$$
P^{S}(x)=b_{0}^{S}-\left(b_{0}^{S}-\theta_{S}\right) \frac{\int_{B\left(x, R_{0}\right)}\left(f^{A}(y, t)+f^{B}(y, t)\right) d y}{N^{*}},
$$

where $N^{*}$ is the local population carrying capacity. Assuming that the detection radius is small, $R_{0} \ll 1$, one can write:

$$
\int_{B\left(x, R_{0}\right)}\left(f^{A}(y, t)+f^{B}(y, t)\right) d y=\pi R_{0}^{2}\left(f^{A}(x, t)+f^{B}(x, t)\right)+O\left(R_{0}^{6}\right)
$$

Therefore, ignoring the higher order terms in $R_{0}$, the number of new particles of type-S created at location $x$ during a time $d t$ can be approximated by :

$$
f^{S}(x, t)\left(b_{0}^{S}-\left(b_{0}^{S}-\theta_{S}\right) \frac{f^{A}(x, t)+f^{B}(x, t)}{f^{*}}\right) d x d t
$$

where $f^{*}=\frac{N^{*}}{\pi R_{0}^{2}}$. Similarly for the death process, the number of type-S particles which are destroyed during time $d t$ can be approximated by:

$$
f^{S}(x, t)\left(d_{0}^{S}-\left(d_{0}^{S}-\theta_{S}\right) \frac{f^{A}(x, t)+f^{B}(x, t)}{f^{*}}\right) d x d t .
$$

Introducing these source terms into Eqs 11 and dropping the bars, one obtains:

$$
\left\{\begin{array}{l}
\partial_{t} f^{A}=D^{A} \Delta_{x} f^{A}+\nabla_{x} \cdot\left(f^{A} \nabla_{x}\left(\bar{\Phi}^{A A} * f^{A}\right)\right)+\nabla_{x} \cdot\left(f^{A} \nabla_{x}\left(\bar{\Phi}^{A B} * f^{B}\right)\right)+\nu^{A} f^{A}\left(1-\frac{f^{A}+f^{B}}{f^{*}}\right) \\
\partial_{t} f^{B}=D^{B} \Delta_{x} f^{B}+\nabla_{x} \cdot\left(f^{B} \nabla_{x}\left(\bar{\Phi}^{B B} * f^{B}\right)\right)+\nabla_{x} \cdot\left(f^{B} \nabla_{x}\left(\bar{\Phi}^{B A} * f^{B}\right)\right)+\nu^{B} f^{B}\left(1-\frac{f^{A}+f^{B}}{f^{*}}\right)
\end{array}\right.
$$

where $\nu^{S}=b_{0}^{S}-d_{0}^{S}$.

Remarks:

- We do not normalize the cell distributions 10 by $N_{A}, N_{B}$ in order to keep the information of the total number of cells. The total mass is essential at the cell level to determine the cell birth process. If one would like to study the asymptotic limit $N_{A}, N_{B} \rightarrow \infty$, one would have to normalize the empirical distribution by the number of cells and consider the limit $R \rightarrow 0$ (see [3]).

- It should be noted that the PDE model with non-normalized densities differs from the one derived in [3], in which the number of links had to be kept proportional to the number of cells to enable the limiting procedure to be well-defined. Here, because the PDE model is kept at the level of (large but) finite number of particles, such assumption is not needed for the derivation.

\section{Stability ANALYSis}

We first note that the non-trivial constant (homogeneous) steady states $\bar{f}^{A}, \bar{f}^{B}$ such that

$$
\bar{f}^{A}+\bar{f}^{B}=f^{*},
$$

are solutions of the macrocopic equation given by Eqs. 12). In order to assess the stability of the homogeneous steady states, we will perform a linear stability analysis using a perturbative approach. We will briefly comment on the cases $\bar{f}^{A}=0$ or $\bar{f}^{B}=0$ (also steady-states of System (12)), but will mostly focus on the non-extinction cases $\left(\bar{f}^{A}, \bar{f}^{B} \neq 0\right)$. 


\subsection{Stability of homogeneous steady states}

In order to perform a linear stability analysis, we use perturbation terms and Fourier transform as done in 3. To this aim, we write:

$$
f^{A}=\bar{f}^{A}+f_{\epsilon}^{A}, \quad f^{B}=\bar{f}^{B}+f_{\epsilon}^{B},
$$

and will ommit the $\epsilon$ for the sake of simplicity. Linearizing Eqs. 12 and taking the Fourier transform, we obtain:

$$
\partial_{t}\left(\begin{array}{c}
\hat{f}^{A} \\
\hat{f}^{B}
\end{array}\right)(y, t)=M(y)\left(\begin{array}{c}
\hat{f}^{A} \\
\hat{f}^{B}
\end{array}\right)(y, t)
$$

where $M$ reads:

$$
M(y)=\left(\begin{array}{cc}
-|y|^{2}\left(2 \pi \bar{f}^{A} \hat{\Phi}^{A A}(y)+D_{A}\right)-\nu^{A} \frac{\bar{f}^{A}}{f^{*}} & -|y|^{2} 2 \pi \bar{f}^{A} \hat{\Phi}^{A B}(y)-\nu^{A} \frac{\bar{f}^{A}}{f^{*}} \\
-|y|^{2} \bar{f}^{B} \hat{\Phi}^{B A}(y)-\nu^{B} \frac{\bar{f}^{B}}{f^{*}} & -|y|^{2}\left(2 \pi \bar{f}^{B} \hat{\Phi}^{B B}(y)+D_{B}\right)-\nu^{B} \frac{\bar{f}^{B}}{f^{*}}
\end{array}\right) .
$$

Note that system (14) has been obtained neglecting the perturbation terms of order two.

In the general case, the homogeneous steady states will be stable only if the real part of the eigenvalues of the matrix $M(y)$ are both negative, otherwise it will be unstable. Since we know that $\operatorname{det}(M(y))=\lambda_{1} \cdot \lambda_{2}$ and $\operatorname{tr}(M(y))=\lambda_{1}+\lambda_{2}$, with $\lambda_{1}(y), \lambda_{2}(y)$ eigenvalues, the stability occurs only if:

$$
\operatorname{det}(M(y))>0 \text { and } \operatorname{tr}(M(y))<0 .
$$

We first compute the trace of matrix $M(y)$ :

$$
\operatorname{tr}(M(y))=-|y|^{2}\left(2 \pi \bar{f}^{A} \hat{\Phi}^{A A}(y)+D_{A}\right)-\nu^{A} \frac{\bar{f}^{A}}{f^{*}}-|y|^{2}\left(2 \pi \bar{f}^{B} \hat{\Phi}^{B B}(y)+D_{B}\right)-\nu^{B} \frac{\bar{f}^{B}}{f^{*}} .
$$

In what follows, we consider the following assumption as in [3] for the parameters:

Hypothesis 1. The intraspecies (or homotypic) links generate repulsive interactions, i.e $\kappa^{A A}>0$ and $\kappa^{B B}>0$.

We can easily note that under Hypothesis 1 , the trace is always negative. Then we compute the determinant of matrix $M(y)$ :

$$
\begin{aligned}
\operatorname{det}(M(y)) & =|y|^{4}\left[\left(\bar{f}^{A} 2 \pi \hat{\Phi}^{A A}+D_{A}\right)\left(\bar{f}^{B} 2 \pi \hat{\Phi}^{B B}+D_{B}\right)-\bar{f}^{A} \bar{f}^{B} 4 \pi^{2} \hat{\Phi}^{A B} \hat{\Phi}^{B A}\right]+ \\
& +|y|^{2}\left[\nu^{B} \frac{\bar{f}^{B}}{f^{*}}\left(\bar{f}^{A} 2 \pi \hat{\Phi}^{A A}+D_{A}-\bar{f}^{A} 2 \pi \hat{\Phi}^{A B}\right)+\nu^{A} \frac{\bar{f}^{A}}{f^{*}}\left(\bar{f}^{B} 2 \pi \hat{\Phi}^{B B}+D_{B}-\bar{f}^{B} 2 \pi \hat{\Phi}^{B A}\right)\right] .
\end{aligned}
$$

The first part with term in $|y|^{4}$ is exactly the determinant computed in [3] without logistic term. The second one is due to the introduction of logistic growth.

As the Trace of $M$ is always negative under Hypothesis 1 . one observes that the constant steady states will be unstable if $\Delta(M)<0$ only. From Eq. (17), it is clear that the interspecies potential intensities controlled by parameters $\kappa^{A B}, \kappa^{B A}$ must be large enough to allow the determinant to be negative. In order to quantify the relative importance of interspecies potential parameters compared to the others (diffusion intensities, growth rates, intraspecies potential intensities), we introduce a parameter $s \in \mathbb{R}^{+}$such that $\kappa^{A B}=s \widetilde{\kappa}^{A B}, \kappa^{B A}=s \widetilde{\kappa}^{B A}$. We consider the following hypothesis on heterotypic interactions:

Hypothesis 2. The interspecies (or heterotypic) links interactions are both repulsive, i.e $\kappa^{A B}>0$ and $\kappa^{B A}>0$. 
Following the same workflow and approach of [3], we can conclude that there exists a critical value $s_{L}^{*}$ for $s$ beyond which the homogeneous steady-states are unstable (corresponding to segregation between the two families):

$$
s_{L}^{*}=\frac{\left(24 D_{A}+c^{A A} \bar{f}^{A}\right) \nu^{B} \bar{f}^{B}+\left(24 D_{B}+c^{B B} \bar{f}^{B}\right) \nu^{A} \bar{f}^{A}}{\nu^{B} \bar{f}^{B} c^{A B} \bar{f}^{A}+\nu^{A} \bar{f}^{A} c^{B A} \bar{f}^{B}},
$$

with $c^{S S}=\frac{2 \pi \kappa^{S S} \nu_{c}^{S S} R^{4}}{\nu_{c}^{S S}+\nu_{d}^{S S}}$ and $c^{S T}=\frac{2 \pi s \widetilde{\kappa}^{S T} \nu_{c}^{S T} R^{4}}{\nu_{c}^{S T}+\nu_{d}^{S T}}, S \neq T \in\{A, B\}$.

Note that in the case of the extinction of one population $\left(\bar{f}^{A}=0\right.$ or $\left.\bar{f}^{B}=0\right)$, the model reduces to a onespecies diffusion equation, which converges in time towards a homogeneous distribution of the survivor species (stable homogeneous state). In what follows, the analysis is carried out outside of this particular regime (i.e for $\bar{f}^{A} \neq 0$ and $\left.\bar{f}^{B} \neq 0\right)$.

\subsection{Characterization of the steady-states}

It is noteworthy that the system's segregation ability depends on the mass ratio of the two families. Indeed, the critical value $s_{L}^{*}$ for which the two-particle system departs from the homogeneous distributions $\bar{f}^{A}, \bar{f}^{B}$ depends on their initial relative ratio. Intuitively, this corresponds to the fact that the amount of mechanical forces exerted by a cell type on the other one must account for its relative mass compared to the other family, for the system to enable cell segregation. In order to give more insights into this phenomenon, we here characterize the stability of the steady states as function of their mass distribution, by studying the influence of the value of $s_{L}^{*}$ as function of the masses $\bar{f}^{A}, \bar{f}^{B}$.

Using $\bar{f}^{B}=f^{*}-\bar{f}^{A}$ we rewrite $s_{L}^{*}$ as:

$$
s_{L}^{*}=\frac{\left(\nu^{B} c^{A A}+\nu^{A} c^{B B}\right) \bar{f}^{A}\left(f^{*}-\bar{f}^{A}\right)+\left(24 D_{B} \nu^{A}-24 D_{A} \nu^{B}\right) \bar{f}^{A}+24 D_{A} \nu^{B} f^{*}}{\bar{f}^{A}\left(f^{*}-\bar{f}^{A}\right)\left(\nu^{B} c^{A B}+\nu^{A} c^{B A}\right)},
$$

It is easy to check that under Hypothesis 1 and Hypothesis $2 \lim _{\bar{f}^{A} \rightarrow f^{*}} s_{L}^{*}\left(\bar{f}^{A}\right)=+\infty$ and $\lim _{\bar{f}^{A} \rightarrow 0} s_{L}^{*}\left(\bar{f}^{A}\right)=+\infty$, meaning that the states corresponding to one dominant population are always stable (see previous remark).

We are looking then for the minimum of function $s_{L}^{*}$, i.e the zero points of $\frac{\partial s_{L}^{*}\left(\bar{f}^{A}\right)}{\partial \bar{f}^{A}}$. After computation we find only one minimum $\bar{f}_{m}^{A}$ in $\left[0, f^{*}\right]$ :

- If $D_{A} \nu^{B}-\nu^{A} D_{B}=0$, the minimum is $\bar{f}_{m}^{A}=\frac{f^{*}}{2}$

- If $D_{A} \nu^{B}-\nu^{A} D_{B} \neq 0$, the minimum is given by:

$$
\bar{f}_{m}^{A}=\frac{f^{*}\left(D_{A} \nu^{B}-\sqrt{D_{A} D_{B} \nu^{B} \nu^{A}}\right)}{D_{A} \nu^{B}-\nu^{A} D_{B}} .
$$

The minimum of function $s_{L}^{*}$ corresponds to the least stable steady state, i.e the steady state which requires the least mechanical efforts to enable segregation between the two species. When the two populations have the same ratio between diffusion and growth, the least stable configuration is the symmetric one. In Figure 2 we report the plot of $s_{L}^{*}$ as a function of $f^{A}$ in the case $D_{A} \nu^{B}-\nu^{A} D_{B} \neq 0$ (blue curve) and $D_{A} \nu^{B}-\nu^{A} D_{B}=0$ (orange curve). As mentioned before, we can see that for given values of the parameters, there exist stable steady states corresponding to the case of a dominant population. For both set of parameters, we can observe a large plateau value meaning that a large number of steady states are unstable beyond the same value of $s$.

\subsection{Impact of the logistic growth on segregation}

It is noteworthy that in the model without logistic growth mass is conserved, i.e the homogeneous state only depends on the initial condition, while with logistic growth, the equilibrium state is determined both by the 


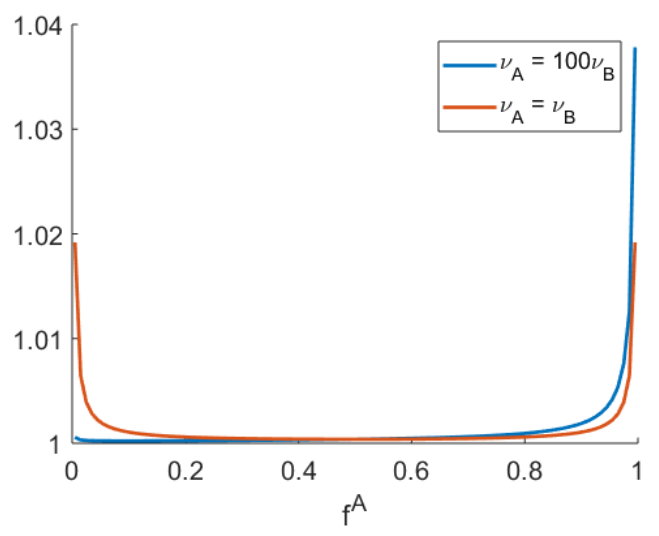

Figure 2. Value of $s_{L}^{*}$ as function of $\bar{f}^{A}$, for $f^{*}=1, D_{A}=D_{B}, \nu_{A}=100 \nu_{B}$ (blue curve), $\nu_{A}=\nu_{B}$ (orange curve).

initial condition and by the birth/death parameters. Without logistic growth, we report the following critical value $s_{C}^{*}$ of $s$ beyond which the system produces segregation (see $[3]$ ):

$$
s_{C}^{*}=\left[\frac{576}{c^{A B} c^{B A} f^{A} f^{B}}\left(D_{A}+\frac{c^{A A} f^{A}}{24}\right)\left(D_{B}+\frac{c^{B B} f^{B}}{24}\right)\right]^{\frac{1}{2}} .
$$

As previously noted, both critical values $s_{C}^{*}, s_{L}^{*}$ are markers of instability and we will discuss some simulations to compare them. As remarked in [3], as diffusion and intra-species repulsion tend to homogenize the system, then the inter-species repulsion forces must be large enough to compensate these phenomena and produce segregation. Thanks to the stability analysis, we can observe and conclude that the logistic growth can either support or repress cell segregation, depending on the choice of the parameters. The segregation is viewed as a breakdown of stability caused by changes in the parameters which characterize the system.

In the next section we will discuss some numerical simulations on the agent-based model to explore numerically the results provided by the stability analysis. If not otherwise stated, the parameters of the simulations are the ones summarized in Table 1. We will explore different parameter regimes:

- case $s_{C}^{*}<s_{L}^{*}$ : If $s$ is such that $s<s_{C}^{*}$, both model should converge towards a homogeneous state (mixing of the two families). If $s \in\left(s_{C}^{*}, s_{L}^{*}\right)$ the model without growth should segregate the two cell types (instability of the homogeneous state), while the model with logistic growth should maintain a homogeneous state. Finally for $s>s_{L}^{*}$, cell segregation should be observed for both models.

- case $s_{C}^{*}>s_{L}^{*}$. Values of $s$ such that $s>s_{C}^{*}$ should produce segregation of the two families for both models, while for $s<s_{L}^{*}$ the system should converge towards homogeneous steady states for both models. For $s$ chosen in the range $\left(s_{L}^{*}, s_{C}^{*}\right)$, the model without growth should create homogeneous state while the model with logistic growth should segregate the two families.

\section{NUMERICAL SCHEME}

We perform numerical simulations of the microscopic model as done in [3] following scheme in [2], on a $2 D$ domain $[-L, L] \times[-L, L]=[-7.5,7.5]^{2}$ with periodic boundary conditions. We set diffusion constants $D_{A}=D_{B}=10^{-4}$ and investigate different values of inter- and intra- species intensities such as $\kappa^{A A}, \kappa^{B B}, \kappa^{A B}=$ $s \tilde{\kappa}^{A B}, \kappa^{B A}=s \tilde{\kappa}^{B A}$. For each equation of system (1) we have the following time discretization:

$$
X_{i}^{n+1}=X_{i}^{n}-\nabla_{X_{i}} W\left(X^{n}\right) \Delta t^{n}+\sqrt{2 D \Delta t^{n}} \mathcal{N}(0,1)
$$




\begin{tabular}{lcc}
\hline Parameters & Description & Values \\
\hline$L$ & Length related to periodic square demain & 7.5 \\
$N_{A}$ & Number of A-type particles & 500 \\
$N_{B}$ & Number of B-type particles & 500 \\
$\mu$ & Motility coefficient & 1 \\
$D_{S}$ & Diffusion coefficient & $10^{-4}$ \\
$\kappa^{A B}$ & Interspecies (or heterotypic) interaction intensity & $\mathrm{S}$ \\
$\kappa^{B A}$ & & $s$ \\
$\kappa^{A A}$ & Intraspecies (or homotypic) interaction intensity & 4 \\
$\kappa^{B B}$ & & 1 \\
$R$ & Radius of interaction (link repulsion) & 1 \\
$R_{0}$ & Logistic radius & 1.5 \\
$r$ & Maximal distance of a daughter cell to her parent & 1.5 \\
$N^{*}$ & Local population carrying capacity & $500 \pi R_{0}^{2}$ \\
$\nu^{A}$ & Logistic growth rate for the A cells & $5.1 L^{2}$ \\
$\nu^{B}$ & Logistic growth rate for the B cells & $5.10^{-3}$ \\
\hline
\end{tabular}

TABLE 1. Parameters and values used for the simulations of the microscopic model

$\mathcal{N}(0,1)$ is the normal distribution with mean 0 and standard deviation 1.

The logistic growth is modelled via independent Poisson processes of position-dependent frequencies $\beta_{S}\left(X_{i}\right)$ and $\delta_{S}\left(X_{i}\right)$ for birth and death respectively. The probability of a cell to divide and/or die between time steps $t$ and $t+\Delta t$ is given by:

$$
\begin{cases}\mathbb{P}(\text { cell i divide }) & =1-\exp ^{-\max \left(0, \beta_{S}\left(X_{i}\right) \Delta t\right)} \\ \mathbb{P}(\text { cell i die }) & =1-\exp ^{-\max \left(0, \delta_{S}\left(X_{i}\right)\right) \Delta t},\end{cases}
$$

where the rates $\beta_{S}$ and $\delta_{S}$ are computed thanks to (3). All simulations are performed with parameters $b_{0}^{S}=$ $\frac{4 \nu^{S}}{3}, d_{0}^{S}=\frac{\nu^{S}}{3}$ and $d_{0}^{S}<\theta^{S}=\nu^{S}<b_{0}^{S}$ for $S=A, B$. Note that these parameters are chosen such that the macroscopic logistic rate $\nu^{S}=b_{0}^{S}-d_{0}^{S}$. For each dividing 'parent' cell $j$, the 'daughter' cell is supposed to be born at a distance randomly chosen in $B\left(X_{j}, r\right)$, where we chose $r=1.5$ from her parent.

Finally, we ensure that only one birth/death event happens between time $t$ and $t+\Delta t$ by choosing the time step $\Delta t \ll \frac{1}{\max \left(\nu^{A}, \nu^{B}\right) N}$, where $N$ is the maximal number of particles between family A and B.

We consider a periodic domain randomly filled with $N_{A}=N_{B}=500$ cells initially and we fix $\kappa^{S T}$ for $S$ and $T$ such as given in table 1. For such values, the critical value $s_{C}^{*}$ of $s$ beyond which the system without logistic growth aggregates is $s_{C}^{*} \approx 2.1$. We consider different logistic rates but we keep the ratio $\frac{\nu^{B}}{\nu^{A}}=10$ such that the critical value $s_{L}^{*}$ of $s$ beyond which the model with logistic growth should produce aggregates is around $s_{L}^{*} \approx 3.8$. For each regime, we therefore explore the cases:

- $\mathrm{s}=1.5$ : both the original model and the one with logistic growth should produce homogeneous steady states

- $\mathrm{s}=2.5$ : the original model should produce an aggregated steady state while the one with logistic growth is expected to produce a homogeneous one.

- $\mathrm{s}=4$ : both the original model and the one with logistic growth should produce aggregated steady states.

In Fig. 3, we show the results of the simulations at time $t=20000$, without logistic growth (left column) and with logistic growth for five different values of $\nu^{B}$ (keeping the ratio $\frac{\nu^{B}}{\nu^{A}}=10$ constant, columns two to six: $\nu^{B}=10^{-3}, 2.10^{-3}, 5.10^{-3}, 10^{-2}, 0.1$ ). For each regime, we consider the cases $s=1.5, s=2.5, s=4$.

As one can observe in Fig. 3 column 1, the microscopic model without logistic growth is in good accordance with the predictions of the stability analysis of the macroscopic model since we observe aggregates for $s=$ 


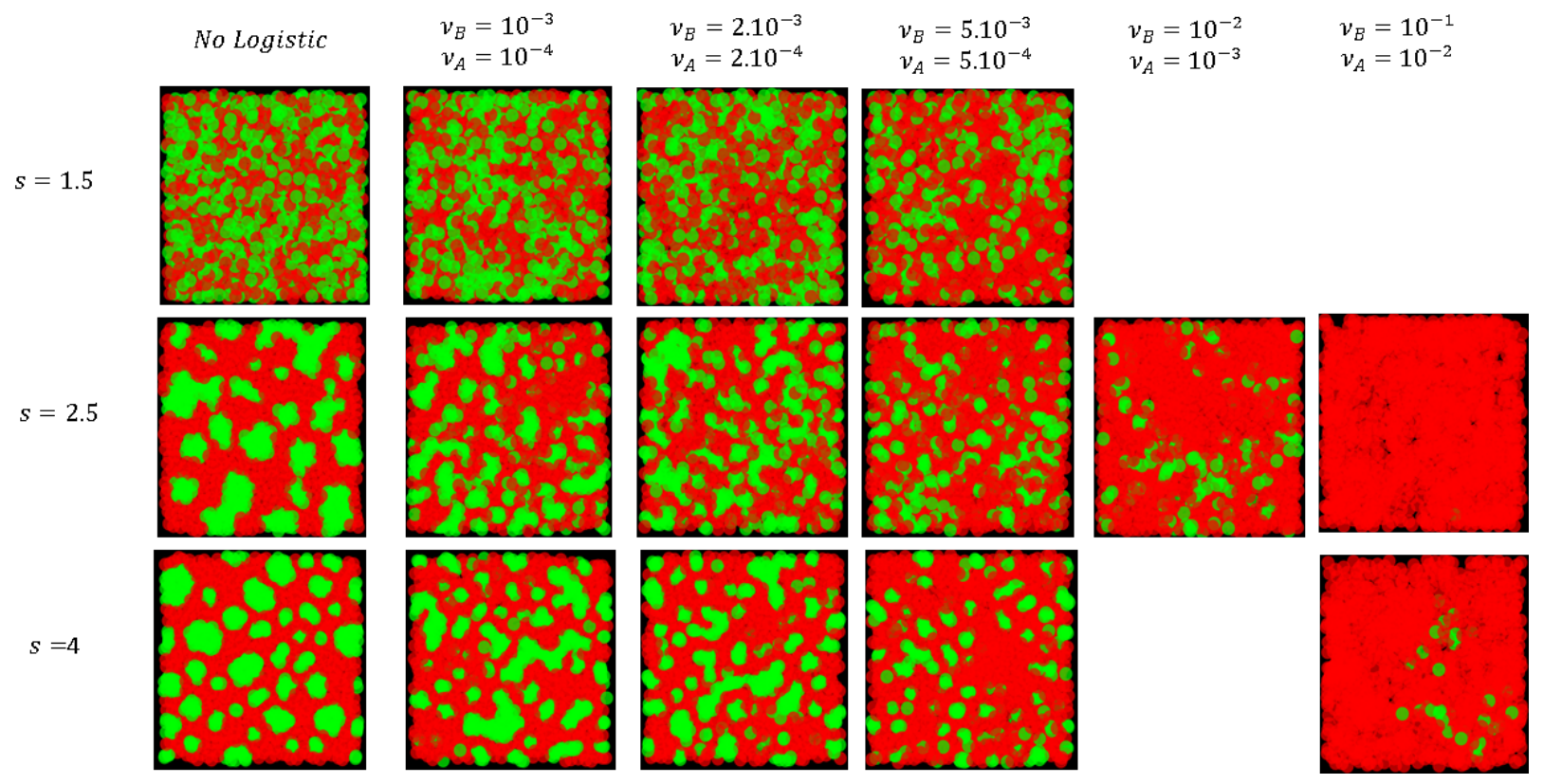

Figure 3. Results of the microscopic model at time $t=20000$, without logistic growth (left column) and with logistic growth for five different values of $\nu^{B}$ (keeping the ratio $\frac{\nu^{B}}{\nu^{A}}=10$ constant, columns two to six: $\left.\nu^{B}=10^{-3}, 2.10^{-3}, 5.10^{-3}, 10^{-2}, 0.1\right)$. For each regime, we consider the cases $s=1.5, s=2.5, s=4$. Cells of family B are represented in green, cells of family A in red.

2.5, 4 and a homogeneous steady-state for $s=1.5$. When activating the logistic growth, one can observe a homogeneous steady-state for $s=1.5$ and aggregated steady states for $s=4$ provided the frequencies of the logistic growth are small enough (columns 2-4). For $s=2.5$, a better mixing of the two populations seems to be observed compared to the case without logistic growth (compare line 2, columns 2-4 to column 1), but the system for $s=2.5$ still shows segregation abilities (compare lines 1 and 2). These observations are quantified below. It is noteworthy that for large values of $\nu^{B}$ (columns 5,6), one can observe at time $t=20000$ the extinction of family B. This suggests that a fast logistic growth process can lead to the complete extinction of one specie. Note that this case was excluded from the stability analysis and the analysis close to this equilibrium state will be the subject of future work.

In order to quantify better the aggregation abilities of the system at equilibrium, we follow the steps of [3] and define a quantifier $Q$ which measures the segregation amount between the two families using image processing tools. To this aim, given a RGB numerical image, we define the quantifier $Q$ as the number of mixed red and green pixels (pixels which have non zero red and green components), normalized by $\min \left(P_{g}, P_{r}\right)$ where $P_{g}, P_{r}$ are the total number of pixels with a non zero green component (resp. red component). Thus defined, $Q=1$ describes a homogeneous state (perfect mixing of the two families), while $Q \ll 1$ for separated phases. Note that because of the normalization, $Q$ does not give a measure of the size/shape of the aggregates. This quantifier only enables to distinct between mixed or separated phases.

In Fig. 4. we show the values of $Q$ as function of the time for three different values of the inter-species repulsion intensities $s=1.5$ (green curves), $s=2.5$ (blue curves) and $s=4$ (orange curve), for $N_{A}=N_{B}=500$ initially. For each case, we consider the case with no logistic growth (continuous lines), with logistic growth for $\nu_{B}=10^{-3}, \nu_{A}=10^{-4}$ (round markers) and $\nu_{B}=5.10^{-3}, \nu_{A}=5.10^{-4}$ (diamond markers). As one can observe, the value of $Q$ decreases as $s$ increases for all the regimes considered, with $Q \approx 1$ for $s=1.5$ (green curves) and independently of the activation of the logistic growth. For $s=2.5$ (blue curves), we observe that the value of $Q$ 


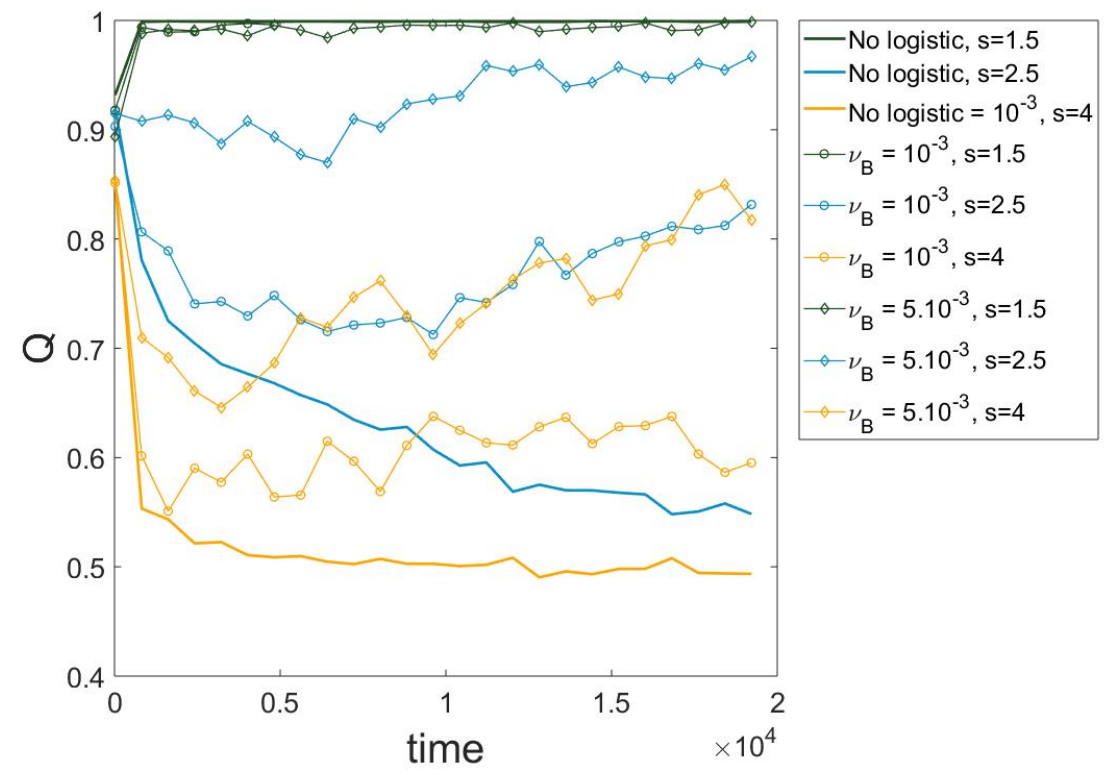

Figure 4. Values of the quantifier $Q$ as function of time for $N_{A}=N_{B}=500$ cells initially, for three different values of the inter-species repulsion $s=1.5$ (green curves), $s=2.5$ (blue curves) and $s=4$ (orange curve), for $N_{A}=N_{B}=500$ initially. For each case, we consider the case with no logistic growth (continuous lines), with logistic growth for $\nu_{B}=10^{-3}, \nu_{A}=10^{-4}$ (round markers) and $\nu_{B}=5.10^{-3}, \nu_{A}=5.10^{-4}$ (diamond markers).

increases as we increase the logistic growth rates, and we recover the predicted homogeneization of the logistic growth for sufficiently large values of $\nu_{B}, \nu_{A}$. If the logistic growth rate is too small $\left(\nu_{B}=10^{-3}, \nu_{A}=10^{-4}\right)$, the system still shows some clustering for $s=2.5$ and $N=500$ (see the blue curve with round markers). For $s=4$ (orange curves), the system produces segregated patterns independently on the logistic growth, as predicted by the stability analysis.

We note that increasing the total number of particles $N_{A}+N_{B}$ (and adapting the parameter $N^{*}$ such that $N_{A}+N_{B}$ corresponds to the equilibrium number of particles) leads to better correspondence between the numerical results and the states predicted by the stability analysis in the macroscopic model. As an illustration, we show in Fig. 5 the case $\nu^{B}=5 \cdot 10^{-3}, \nu^{A}=5 \cdot 10^{-4}$ for $N=500$ and $N=1000$, where the structures observed at $t=20000$ for $s=2.5$ seem to be closer to a homogeneous state for $N=1000$ than for $N=500$. In Fig. 6, we show the values of $Q$ with $N_{A}=N_{B}=1000$ particles initially, for three different values of $s: s=1.5$ (green curves), $s=2.5$ (blue curves), $s=4$ (orange curves) as function of the time without logistic growth (continuous lines) and with logistic growth for $\nu_{B}=5 \cdot 10^{-3}, \nu_{A}=5 \cdot 10^{-3}$ (diamond). By comparing the diamond marked curves of Fig. 6 to the ones of Fig. 4 one can observe a better prediction and stability of the patterns in time for $N=1000$ compared to $N=500$.

Finally in Fig. 7. we show the evolution of the normalized number of cells $\frac{N_{A}}{N_{A}+N_{B}}$ (black curves) and $\frac{N_{B}}{N_{A}+N_{B}}$ (colored curves) as function of the time for $\nu_{B}=10^{-3}$ and $\nu_{A}=10^{-4}$, and different values of $s$ : (I) for $s=1.5$, (II) for $s=2.5$ and (III) for $s=4$. As one can observe in Fig. 7, in the stable cases $s=1.5$ and $s=2.5$, the fraction of cells reaches the equilibrium value corresponding to the predicted distribution given by Eq. (20). For $s=1.5,2.5$, the system stabilizes around $35 \%$ of type B cells and $65 \%$ of type A cells, while in the unstable case $s=4$ the number of cells seems to oscillate around the initial ratio $50 \%$ for each family. Note that these large stochastic oscillations around the mean could be analogue to the ones reported in [5]. In [5], the authors report stochastic fluctuations in a non-spatial model of two competing species submitted to a logistic 


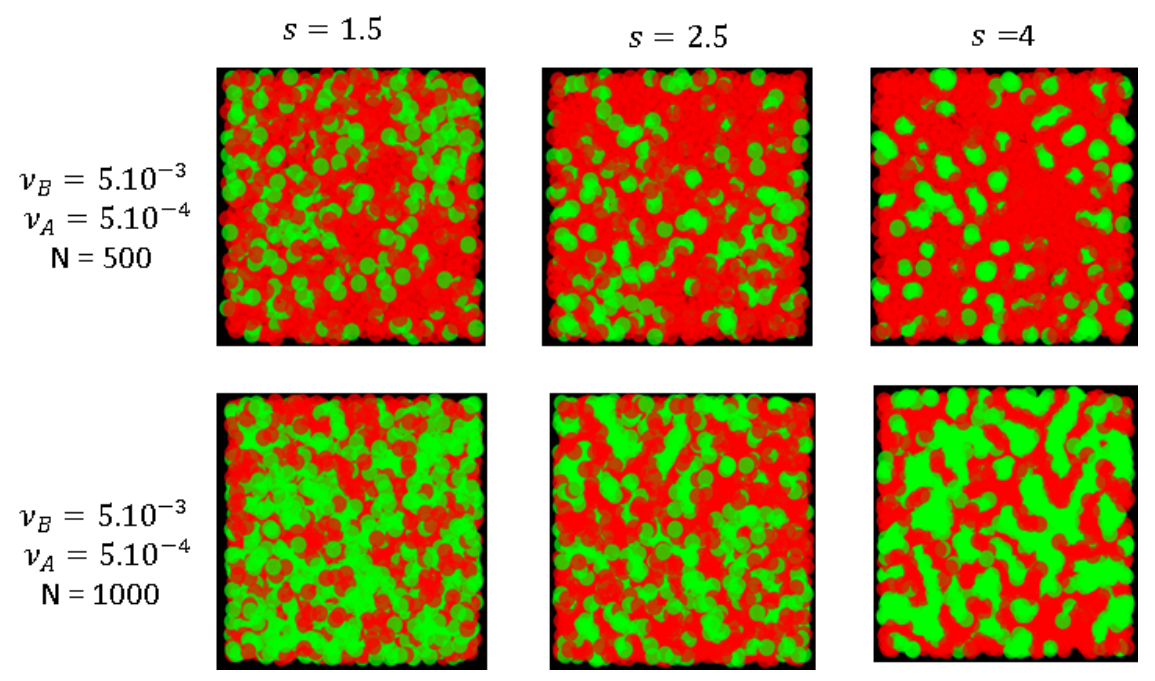

Figure 5. Case $\nu^{B}=5.10^{-3}, \nu^{A}=5 \cdot 10^{-4}$ for $N=500$ (top line) and $N=1000$ (bottom line

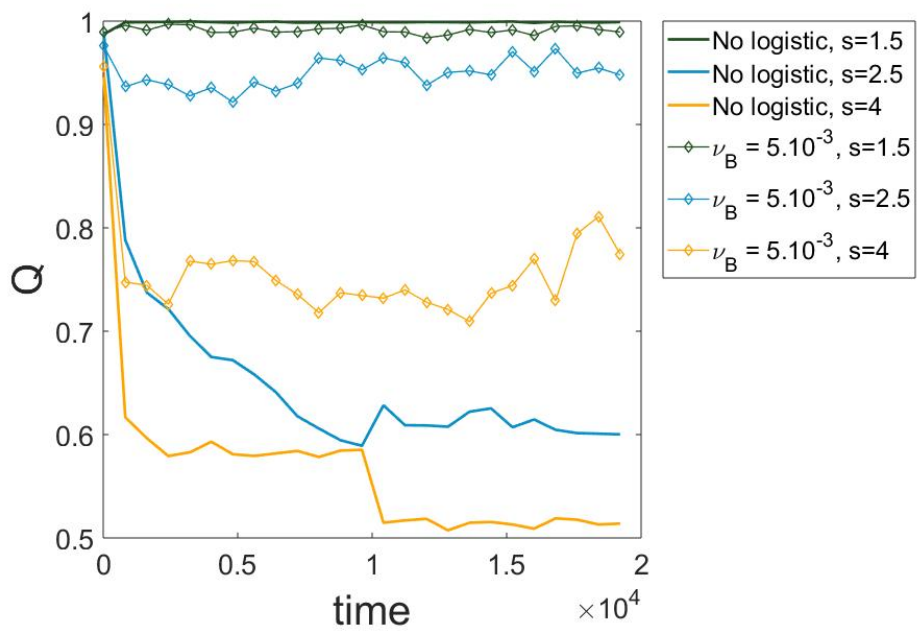

Figure 6. Values of the quantifier $Q$ as function of time for $N_{A}=N_{B}=1000$ cells initially, for three different values of the inter-species repulsion $s=1.5$ (green curves), $s=2.5$ (blue curves) and $s=4$ (orange curve), for $N_{A}=N_{B}=500$ initially. For each case, we consider the case with no logistic growth (continuous lines), and with logistic growth for $\nu_{B}=5.10^{-3}, \nu_{A}=5.10^{-4}$ (diamond markers).

growth. They show that giant fluctuations (the variance being of order of the mean squared) in the number of individuals are obtained if the growth rates of the two families are of the same order, and that in this regime the deterministic equation must be abandoned and a stochastic treatment used instead. By introducing spatial mechanical interactions, we believe that the logistic regime, for which fluctuations are expected, is shifted and plays a role when the logistic growth balances the mechanical forces. Here, even for $\nu_{B}=10 \nu_{A}$, we still observe fluctuations in the number of cells when we are slightly after the transition value for the mechanical interactions. 


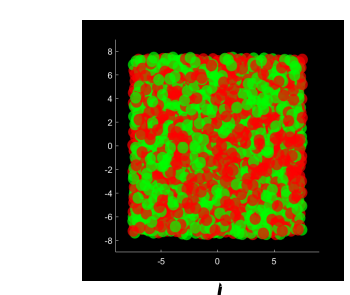

(I)
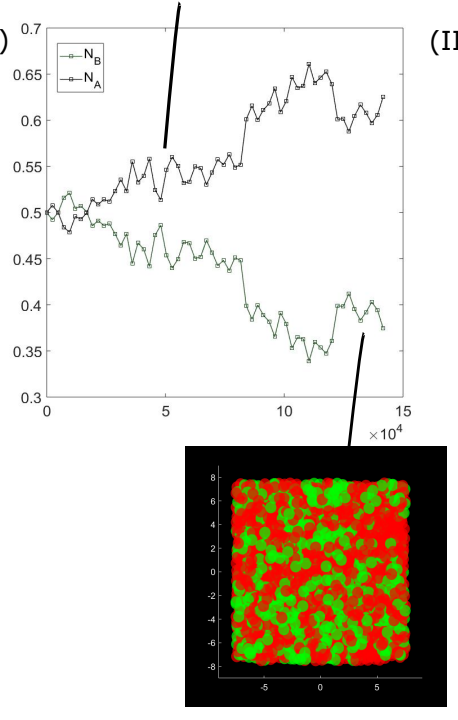

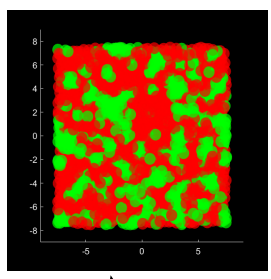

(II)

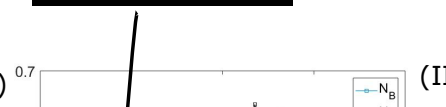

(III)

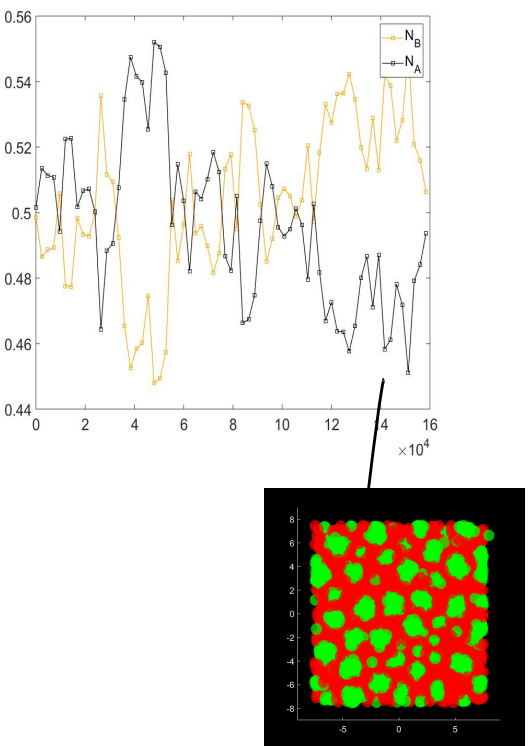

FIgURE 7. Evolution of the normalized number of cells of each family $\frac{N_{A}}{N_{A}+N_{B}}$ (black curves) and $\frac{N_{B}}{N_{A}+N_{B}}$ (colored curves) as function of the time for $\nu_{B}=10^{-3}$ and $\nu_{A}=10^{-4}$ and different values of $s$ : (I) for $s=1.5$, (II) for $s=2.5$ (stable cases) and (III) for $s=4$ (unstable case).

\section{Conclusion}

We have proposed a theoretical and numerical study of a cell division process in a cell aggregation model. The modeling is based on a multiscale approach, from a microscopic model to a macroscopic description of a system of particles interacting through a dynamical network. The model describes point particles with local cross-links modeled by springs that are randomly created and destructed. In the mean field limit, the link density distribution becomes a local function of the particle distribution density, evolving on a slow time scale through a McKean-Vlasov equation. We introduced the cell division process through a logistic growth on the macroscopic model and a birth-death process at the microscopic scale.

The linear stability analysis of the macroscopic model gives access to a criterion on the ratio between heterotypic and homotypic repulsion to ensure the formation of clusters. This criterion involves the logistic growth rate and it is compared to the case of a population with fixed size. Interestingly, the theoretical stability analysis of the macroscopic model showed that introducing a logistic growth term in this cell repulsion-diffusion model can either repress or promote segregation. Indeed, we have shown that the size of the parameter zone for which the homogeneous state is stable depends on the logistic growth parameters, and it can be increased or decreased by choosing the parameters appropriately. The mechanisms can be summarized as follows: Consider a system of two types of particles where heterotypic repulsion forces dominate homotopic forces and diffusion, such that we observe the segregation of the two families in the case of populations of fixed sizes. Close to the transition zone (where the heterotypic forces are close to the critical value), the system can be homogenized by 
introducing a logistic term where the compressed family cell renewal is larger than the one of the predominant family. Enabling the clustered cells to be renewed at the border of the clusters faster than the compressing family leads to a spread out of the clustered family and the overall system converges towards a homogeneous (mixed) state.

In order to validate the theoretical analysis, we performed numerical simulations of the microscopic model which showed a good correspondence with the analysis of the macroscopic model, provided that the parameters are chosen in the right regime. The numerical results showed that for sufficiently large initial number of cells and sufficiently slow logistic growth compared to the time scale of the mechanical interactions, we could recover the homogenization via logistic growth as foreseen by the stability analysis. In time, the system converged towards the predicted value of cell distributions, which showed that the macroscopic model is a good approximation of the microscopic dynamics as the number of cells increases.

Since this work was a first attempt to introduce a logistic term -both at the microscopic/stochastic level and at the macroscopic one- in a system of mechanically interacting particles, several questions remain unanswered and require further investigations. For instance, from a theoretical viewpoint, the rigorous derivation of the macroscopic model is still an open problem. This will require precise estimates depending on the size and number of cells. On the numerical viewpoint, more work is needed to better understand the interplay between the mechanical interactions and the logistic growth in the regime of fast logistic growth. A deeper parametric analysis is needed to better capture the link between the stochastic growth process and its deterministic approximation. Future works will aim to perform the numerical comparison between the macroscopic and microscopic model.

\section{ACKNOWLEDGEMENTS}

The work presented in this paper is the result of a fruitful collaboration that took place during the CEMRACS 2018, organized by Vincent Calvez (Institut Camille Jordan, CNRS, Univ. Lyon 1) Celine Grandmont (Equipe projet Reo et LJLL, Inria Paris et Sorbonne Univ), Eva Locherbach (Departement de Mathematiques, Univ. de Cergy Pontoise), Clair Poignard (Equipe Projet Monc et IMB, Inria Bordeaux et Univ. de Bordeaux), Magali Ribot (Institut Denis Poisson, Univ. d'Orleans), Nicolas Vauchelet (Laga, Univ. Paris 13). This work was supported by the European Research Council (ERC) under the European Union s Horizon 2020 research and innovation program (grant agreement No 740623).

\section{REFERENCES}

[1] J. Barré, P. Degond, E. Zatorska. Kinetic theory of particle interactions mediated by dynamical networks. SIAM MMS (2017) 15(3): 1294-1323.

[2] J. Barré, J.A. Carrillo, P. Degond, D. Peurichard, E. Zatorska. Particle interactions mediated by dynamical networks: assessment of macroscopic description. Nonlinear sci (2017). https://doi.org/10.1007/s00332-017-9408-z.

[3] J. Barré, P. Degond, D. Peurichard, E. Zatorska. Modelling pattern formation through differential repulsion (2019), arXiV: https://arxiv.org/pdf/1906.00704.pdf

[4] J. A. Carrillo, A. Chertock, Y. Huang. A finite-volume method for Nonlinear Nonlocal Equations with a Gradient Flow Structure. Commun. Comput. Phys. (2015), 17(1):233-258.

[5] B. Houchmandzadeh. Giant fluctuations in logistic growth. Physical Review E : Statistical, Nonlinear, and Soft Matter Physics, American Physical Society, 2018, 98, pp.042118.

[6] H. B. Taylor, A. Khuong A, Z. Wu, Q. Xu, R. Morley, L. Gregory, A. Poliakov, WR Taylor, DG Wilkinson. Cell segregation and border sharpening by Eph receptor-ephrinmediated heterotypic repulsion. J. R. Soc. Interface 14: 20170338. (2017)

[7] N. Tkachenko, J. D. Weissmann, W. P. Petersen, G. Lake, C. P. E. Zollikofer, S. Callegari. Individual-based modelling of population growth and diffusion in discrete time. PLoS ONE 12(4): e0176101. (2017)

[8] D. A. Birch, W. R. Young A master equation for a spatial population model with pair interactions. Theoretical Population Biology. (2006), 70(1):26-42.

[9] R. Law, D. J. Murrell, and U. Dieckmann. Population growth in space and time: spatial logistic equations. The Ecological Society of America, (2003), 84(1):252-262

[10] H. Risken, Fokker-Planck Equation, The Fokker-Planck Equation: Methods of Solution and Applications, Springer Berlin Heidelberg, (1984), 63-95. 